Sección Básica / Basic

Artículo de investigación / Research paper

\title{
Aspectos morfo-fisiológicos del tracto digestivo en larvas de IV estadio de Bradysia impatiens (Diptera: Sciaridae)
}

\author{
Morphophysiological aspects of the digestive tract in IV stage larvae of Bradysia \\ impatiens (Diptera: Sciaridae)
}

\author{
VICTOR HUGO MARÍN-CRUZ'; SILVIA RODRÍGUEZ-NAVARRO2; JUAN ESTEBAN \\ BARRANCO-FLORIDO ${ }^{3}$; ROBERTO ALEJANDRO TERRÓN-SIERRA ${ }^{4}$; DAVID CIBRIÁN-TOVAR ${ }^{5}$
}

\begin{abstract}
${ }^{1}$ Maestro en Ciencias, Estudiante de Doctorado en Ciencias Agropecuarias, Universidad Autónoma Metropolitana Unidad Xochimilco, CP 04960, Ciudad de México, México, victormarin_@hotmail.es, https://orcid.org/0000-0002-9512-0202. ${ }^{2}$ Doctora en Ciencias, Departamento de Producción Agrícola y Animal, Universidad Autónoma Metropolitana Unidad Xochimilco, CP 04960, Ciudad de México, México, srodnavarro@gmail.com, https://orcid.org/0000-00015070-6819. ${ }^{3}$ Doctor en Ciencias, Departamento de Sistemas Biológicos, Universidad Autónoma Metropolitana Unidad Xochimilco, Ciudad de México, CP 04960, Universidad Autónoma Metropolitana Unidad Xochimilco, CP 04960, Ciudad de México, México, barranco@correo.xoc.uam.mx, https://orcid. org/0000-0002-2022-0479. ${ }^{4}$ Maestro en Ciencias, Departamento de Producción Agrícola y Animal, Universidad Autónoma Metropolitana Unidad Xochimilco, CP 04960, Ciudad de México, México, terronr@correo.xoc.uam.mx, https://orcid.org/0000-0003-4786-9099. ${ }^{5}$ Doctor en Ciencias, División de Ciencias Forestales, Universidad Autónoma Chapingo, Texcoco, CP 56230, Chapingo, Estado de México, México,dcibrian48@gmail.com, https://orcid.org/00000001-6788-0178.
\end{abstract}

\begin{abstract}
Autor para correspondencia: Victor Hugo Marín-Cruz, Maestro en Ciencias, Estudiante de Doctorado en Ciencias Agropecuarias, Universidad Autónoma Metropolitana Unidad Xochimilco, Calzada del Hueso 1100, Col. Villa Quietud, Delegación Coyoacán, CP 04960, Ciudad de México, México, victormarin_@ hotmail.es, https://orcid.org/0000-0002-95120202
\end{abstract}

Citación sugerida / Suggested citation: MARÍN-CRUZ, V. H.; RODRÍGUEZNAVARRO, S.; BARRANCO-FLORIDO, J. E.; TERRÓN-SIERRA, R. A.; CIBRIÁN-TOVAR, D. 2019. Aspectos morfo-físiológicos del tracto digestivo en larvas de IV estadio de Bradysia impatiens (Diptera: Sciaridae). Revista Colombiana de Entomología 45 (2): e7962. https://doi.org/10.25100/socolen.v45i2.7962

Recibido: 29-mar-2018

Aceptado: 27-abr-2019

Publicado: 20-ene-2020

Revista Colombiana de Entomología ISSN (Impreso): 0120-0488

ISSN (En línea): 2665-4385

http://revistacolombianaentomologia.univalle.edu.co/

Open access

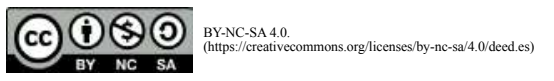

Publicadores / Publishers:

Sociedad Colombiana de Entomología SOCOLEN (Bogotá, D. C., Colombia) http://www.socolen.org.co Universidad del Valle (Cali, Colombia) http://www.univalle.edu.co/

(C) 2019 Sociedad Colombiana de Entomología - SOCOLEN y Universidad del Valle - Univalle
Resumen: La morfología, citología y algunos aspectos fisiológicos del tracto digestivo de larvas de cuarto estadio del siárido Bradysia impatiens se estudiaron utilizando microscopía de campo brillante. Se observó la división del tracto digestivo (estomodeo, mesenterón y proctodeo); las células que lo componen son columnares, regenerativas y endocrinas. Las larvas no presentan buche y la digestión del bolo alimenticio se realiza en el intestino medio; tienen dos ciegos gástricos bien desarrollados, que permiten suponer que existe una gran absorción de nutrientes. La membrana peritrófica es del tipo II, con un tamaño de poro que permite el paso de moléculas de hasta $150 \mathrm{kDa}$ y divide el intestino medio en dos regiones, los espacios endo y ecto peritrófico. Tiene cuatro tubos de Malpighi. El intestino medio posee un gradiente de $\mathrm{pH}$ que va de alcalino en el intestino anterior a neutro en los ciegos gástricos y el intestino posterior. Tiene un mecanismo de recirculación que mejora la digestión y reutilización de enzimas digestivas, que va del espacio endoperitrófico al ectoperitrófico y viceversa. Las características morfofísiológicas mostradas en las larvas de B. impatiens permiten confirmar que son organismos bien adaptados como detritívoros. Además, la membrana peritrófica protege al intestino medio de agentes patógenos.

Palabras clave: Estomodeo, mesenterón, proctodeo, membrana peritrófica, ciegos gástricos, Diptera, Sciaridae.

Abstract: The morphological, cytological and physiological aspects of the digestive tract of the siarid Bradysia impatiens fourth-instar larvae were studied using bright-field microscopy. The division of the digestive tract into the stomodeum (foregut), the mesenteron (midgut) and the proctodeum (hindgut) was observed; the second of which being composed of endocrine, regenerative, column-shaped cells. The larvae did not present a crop; hence digestion of the bolus occurs in the midgut. They have two well-developed gastric caeca, where it can be assumed that great nutrient absorption takes place. The peritrophic membrane is type II, with a pore size that allows the passage of molecules up to $150 \mathrm{kDa}$, and which divides the midgut into two regions: the endoperitrophic and ectoperitrophic spaces. There are four Malpighian tubules present. The midgut has a $\mathrm{pH}$ gradient ranging from mildly alkaline in the foregut to neutral in the caeca and hindgut. It has a recirculation mechanism stretching from the endoperitrophic to the ectoperitrophic space and vice versa, which improves digestion and optimizes enzyme production. The morphophysiological features observed in B. impatiens larvae confirm that these organisms are well-adapted detritivores. Additionally, the peritrophic membrane protects the midgut from pathogenic agents.

Keywords: Stomodeum, mesenteron, proctodeum, peritrophic membrane, gastric caeca, Diptera, Sciaridae.

\section{Introducción}

Bradysia impatiens (Johannsen, 1912) (Diptera: Sciaridae), es un díptero con distribución mundial. En México se le encuentra en invernaderos de ornamentales y viveros forestales (Marín-Cruz et al. 2017). Este díptero ataca las raíces de plántulas de árboles como eucaliptos y coníferas (Menzel et al. 2006; Hurley et al. 2010). Cibrián et al. (2008) reportan daños severos en viveros forestales en 
el centro de México, que causan la pérdida de hasta $30 \%$ de plántulas de coníferas. Las larvas de B. impatiens son detritívoras y consumen materia orgánica en descomposición, hongos y raíces (Marín-Cruz et al. 2015a); es necesario que continuamente ingieran grandes cantidades de alimento. Generalmente las larvas de las especies de insectos detritívoros producen grandes cantidades de enzimas proteolíticas que hidrolizan los nutrientes que están en su alimento (Sharma et al. 1984). Tanto en el cuerpo como en el intestino de las larvas de $B$. impatiens se han aislado especies de hongos de los géneros Verticillium Nees, 1816, Penicillium Link, 1809, Alternaria Nees, 1816, Fusarium Link, 1809, Paecilomyces Bainier, 1907, Aspergillus Micheli, 1729 y Mucor Micheli ex L., 1753 (Marín-Cruz et al. 2015b), algunos de los cuales son fitopatógenos, por los que podrían ser vectores de enfermedades de plantas. Las larvas de B. impatiens ingieren continuamente microorganismos que pueden ser patógenos o sus toxinas, por lo que en el intestino deben de tener mecanismos bien desarrollados que les permitan contrarrestar un posible daño. El intestino de los insectos al igual que en otros animales, es un órgano importante para realizar funciones vitales como la digestión, absorción de nutrientes, regulación de iones y $\mathrm{pH}$, así como la desintoxicación y eliminación de heces (James 2002; Terra y Ferreira 2012). En los insectos el intestino es un órgano esencial para el crecimiento y estado nutricional de las larvas, ya que puede afectar los subsiguientes estados de pupa, adulto y su potencial reproductivo (Chapman 1998). Pese a la importancia agronómica de $B$. impatiens como insecto plaga, no existen estudios de su tracto digestivo. Sin embargo, la anatomía interna y condiciones fisiológicas en el intestino de las larvas se ha investigado en otras especies de dípteros como Ochlerotatus togoi (Theobald, 1907) (Diptera: Culicidae) (Intakhan et al. 2014); "mosca negra" 45 especies (Diptera: Simuliidae) (Kim y Adler, 2009); Lutzomyia longipalpis (Lutz y Neiva, 1912) (Diptera: Psychodidae) (Do Vale et al. 2007); Chrysomia megacephala (Fabricius, 1794) (Diptera: Calliphoridae) (Boonsriwong et al. 2006); Odontosciara Rübsaamem, 1908 (Diptera: Sciaridae) (Cruz y Morelli 1985) y Tipula abdominalis (Say, 1823) (Diptera: Tipulidae) (Sharma et al. 1984).

En este trabajo se describió la anatomía del intestino de larvas de cuarto estadio de $B$. impatiens, el tipo de células que lo componen, el gradiente de $\mathrm{pH}$ en el intestino medio y los ciegos gástricos y el mecanismo de recirculación del bolo alimenticio en el intestino medio, así como la permeabilidad de la membrana peritrófica.

\section{Materiales y métodos}

Insectos. Los insectos adultos se recolectaron con un aspirador manual, en febrero de 2015 en plantas de Pinus montezumae (Lamb) (Pinales: Pinaceae) del vivero forestal de Temamatla, Estado de México (1945'11'N, 99¹1'15”O). La identificación se realizó con las claves taxonómicas de la familia Sciaridae de Mohrig y Menzel (2009), Mohrig et al. (2012), Shin et al. (2012), y Marín-Cruz et al. (2015b). Se estableció una cría en el insectario de la Universidad Autónoma Metropolitana Unidad Xochimilco. Las cámaras de cría consistieron en recipientes de plástico transparente de medio litro de capacidad con tapa. En las tapas se realizó un corte circular de $1,5 \mathrm{~cm}$ de diámetro para introducir los adultos de la $B$. impatiens; se colocaron 10 hembras y dos machos. La dieta consistió de $5 \mathrm{~g}$ de bagazo de caña molido y rodajas de papa. Cada cuatro días se agregaron $40 \mathrm{ml}$ de agua destilada, para conservar la humedad. Las cámaras de cría se mantuvieron a $21^{\circ} \mathrm{C} \pm 2{ }^{\circ} \mathrm{C}$ (Marín-Cruz et al. 2015a).

Descripción anatómica del intestino. Se disecaron 20 larvas en solución Ringer para obtener el sistema digestivo e identificar cada una de las partes (Martínez 2002). Se realizó un corte transversal en el ano de la larva y después se realizaron cortes en la unión del tegumento con la cabeza de la larva (Fig. 1A). Todas las observaciones se realizaron con un microscopio óptico compuesto Zeiss ${ }^{\circledR}$ Axiostar Plus, un estereoscopio Zeiss ${ }^{\circledR}$ Stemi $2000-C$ y una cámara digital Canon $^{\circledR}$ A 620 Power Shot.

Determinación de tipo de células del intestino. Las larvas de cuarto estadio (L4) se fijaron en solución de Bouin durante $48 \mathrm{~h}$, se eliminó el fijador con cuatro lavados en alcohol: $70,90,96$ y $100 \%$ y se incluyeron en parafina Merck $^{\circledR}$. Se realizaron cortes longitudinales y transversales seriados de 5 $\mu \mathrm{m}$ con un micrótomo Spencer ${ }^{\circledR} 820$. Los cortes se tiñeron con la técnica de hematoxilina-eosina (Uría y Mora 1996).

pH del intestino medio y ciegos gástricos. Se utilizaron los indicadores de $\mathrm{pH}$ azul de bromotimol ( $\mathrm{pKa} 7)$, rojo de fenol (pKa 7,9) y azul de timol (pKa 8,2). Cada uno se suministró por separado y se mezcló en una concentración de $0,4 \%(\mathrm{p} / \mathrm{p})$ con $0,5 \mathrm{~g}$ de harina de papa esterilizada. Los experimentos se realizaron con 60 larvas, colocadas individualmente en cajas Petri de $1,5 \mathrm{~cm}$ de diámetro, cada larva se alimentó ad libitum con la mezcla de harina de papa y el colorante indicador.
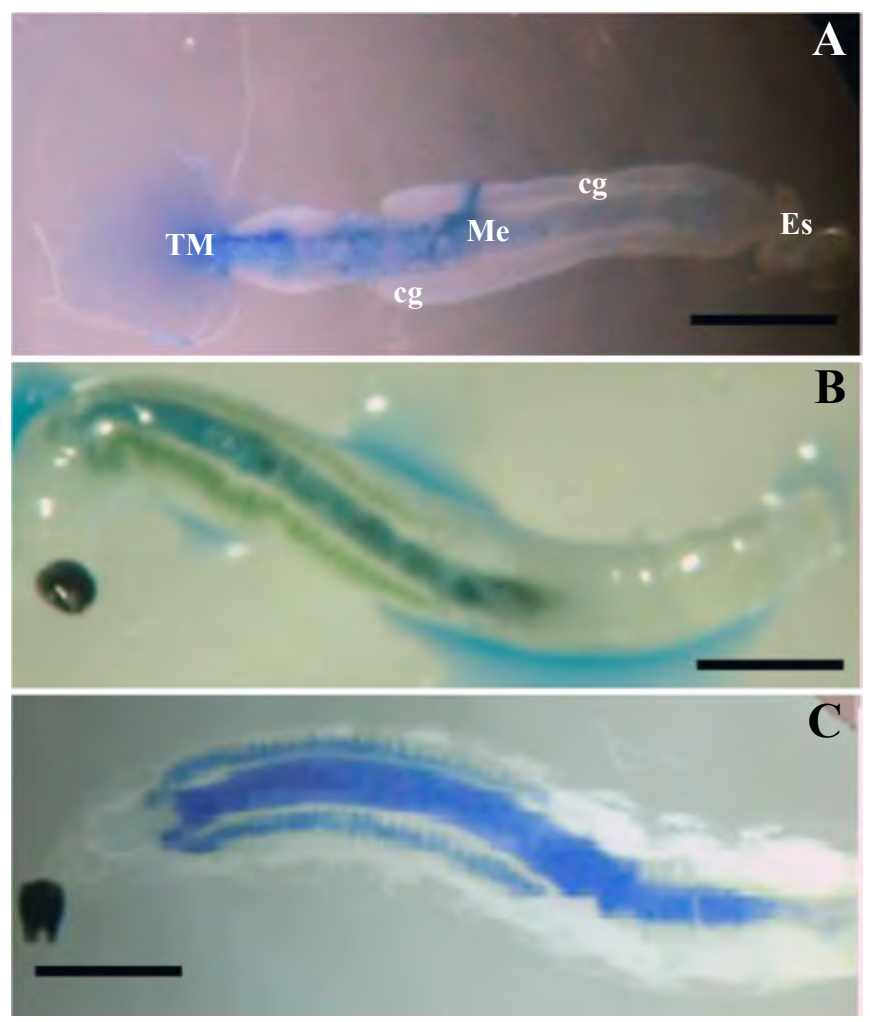

Figura 1. A. Tracto digestivo de larva de cuarto estadio de Bradysia impatiens. B. Estructuras del intestino medio con coloración diferente por el indicador de $\mathrm{pH}$ azul de bromotimol. C. Larva tenida con azul de dextran de 20,000 Da. estomodeo (Es), mesenterón (Me), ciegos gástricos (cg), tubos de Malpighi (TM). Escala: $300 \mu \mathrm{m}$. 
Después de 24 h se estimó el pH en cada región del intestino (Gontijo et al. 1998), para lo cual se compararon los colores dentro de éste con los de soluciones tampón de $\mathrm{pH}$ conocido, preparadas con los mismos indicadores, a partir de las imágenes obtenidas. Las larvas se observaron sin disección, ya que el tegumento larvario es transparente. Se realizaron 20 observaciones para cada indicador de pH (Fig. 1B).

Recirculación del bolo alimenticio. El mecanismo de recirculación (contra corriente) en el intestino medio se determinó en 120 larvas, en grupos de 40, que se ubicaron en cajas Petri de $9 \mathrm{~cm}$ de diámetro. Se alimentaron con harina de papa $(0,1 \mathrm{~g})$ y $150 \mu \mathrm{l}$ azul de dextran de $20.000 \mathrm{Da}$, al 0,05 $\% \mathrm{p} / \mathrm{p}$. Una vez que todo el intestino medio se tiñó con el azul de dextran, las larvas se cambiaron de cajas de Petri, y el colorante, se sustituyó por agua destilada. Se observaron a 15 , 30, 45 min y a 1, 2, 4, 8, 24 y 48 h (Fig. 1C).

Permeabilidad de la membrana peritrófica (MP). Se utilizó azul de dextran de peso molecular de 5.000, 10.000, 20.000 y $500.000 \mathrm{Da}$ (SIGMA-ALDRICH) 0,05\% p/p y como alimento se utilizó $0,04 \mathrm{~g}$ de harina de papa. Cada azul de dextran de diferente peso molecular se suministró a 50 larvas por separado. Se realizaron revisiones con un estereoscopio $\left(\right.$ Zeiss $^{\circledR}$ Stemi 2000-C) cada 15, 30, 60 min y 2, 4, 8, 16, 24 y
48 h. Una vez que el espacio endoperitrófico se teñía del azul de dextran, las larvas se enjuagaban con agua destilada y se colocaban en un recipiente sin colorante, con la finalidad de no saturar el intestino (Fig. 1C).

\section{Resultados}

El tracto digestivo en las L4 de B. impatiens se compone de un tubo, dividido en tres regiones bien definidas: estomodeo, mesenterón y proctodeo (Fig. 1A). Las glándulas salivales, ciegos gástricos y tubos de Malpighi, se proyectan del tubo principal del tracto digestivo.

Intestino anterior o estomodeo. Se compone de boca, faringe, esófago y finaliza en la región anterior del proventrículo. Está revestido internamente con una capa cuticular compuesta de una epicutícula interna y una endocutícula externa con células epiteliales aplanadas. En esta sección el bolo alimenticio tiene un tránsito muy rápido. La faringe es un tubo simple (Figs. 2A-2B). Las dos glándulas salivales se componen de células epiteliales de forma cónica simple y núcleos ovalados, estrechamente empaquetadas y proyectan en su parte apical hacia el lumen central de la glándula, se conectan en la boca y la faringe en el cibarium por medio de un conducto deferente (Figs. 2A, 2B y 2E). También se observa un compuesto,
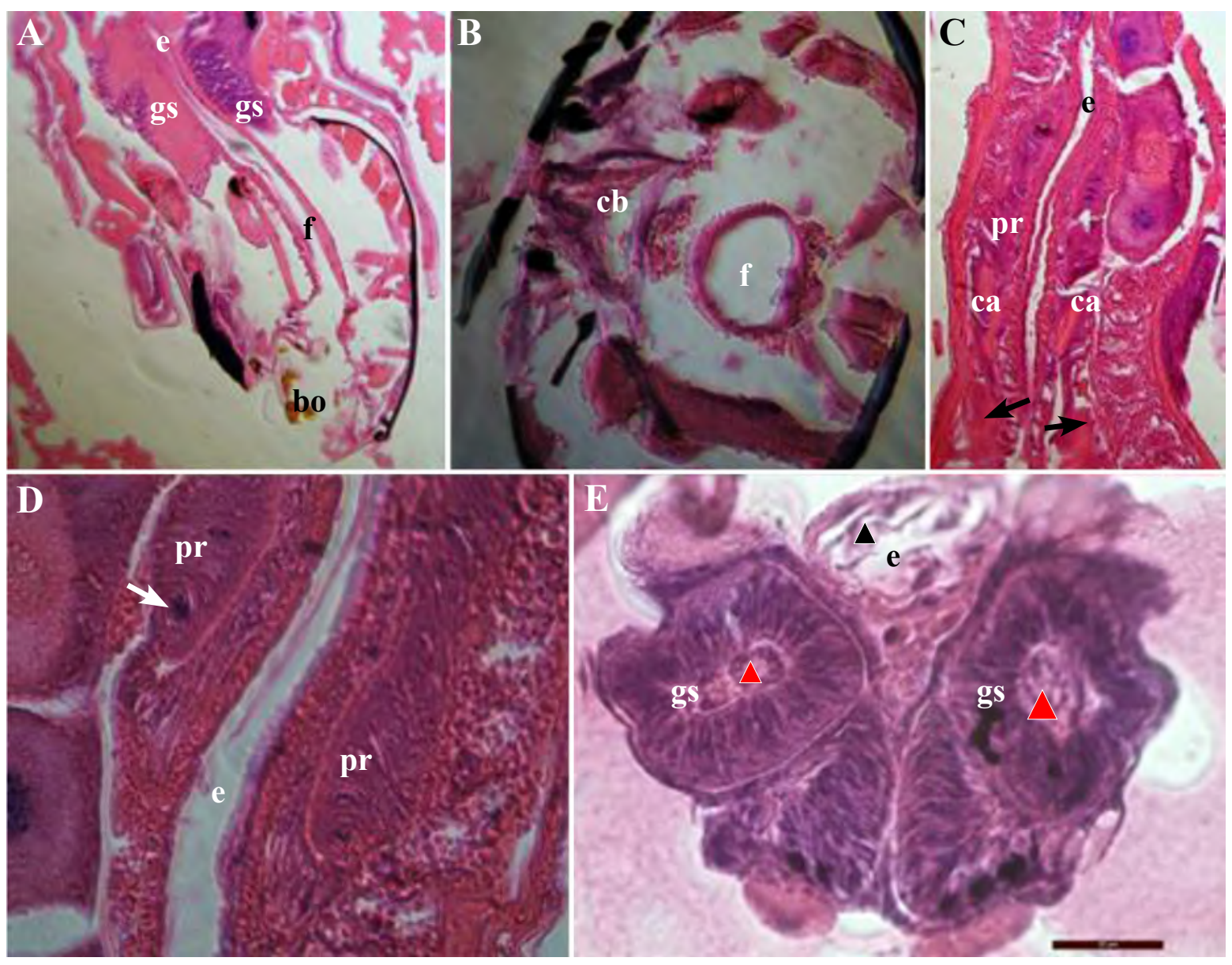

Figura 2. Intestino anterior (estomodeo) de larvas de cuarto instar de Bradysia impatiens. A. Corte longitudinal de cabeza (40x). B. Sección transversal de cabeza (100x). C. Vista longitudinal de proventrículo (40x). D. Corte longitudinal de esófago invaginándose en el proventrículo (100x). E. Glándulas salivales y esofago. Boca (bo), cardia (ca), cibarium (cb), esófago (e), faringe (f), glándulas salivales (gs), proventrículo (pr), secreción de saliva (cabeza de flecha roja); secreción de membrana perotrófica (cabeza de flecha negra); unión del ciego gástrico con el intestino anterior (flecha negra); núcleo celular (flecha blanca). 
posiblemente la secreción de saliva. El esófago es un tubo recto que conecta la faringe con el proventrículo, invaginándose en este último (Figs. 2C-2D), circundado por músculos circulares que rodean este tejido. Las L4 de B. impatiens poseen un proventrículo globoso y el canal alimentario que lo atraviesa es estrecho y circundado. Los cortes histológicos del proventrículo mostraron que esta estructura se compone de dos regiones, una con tejido característico del intestino anterior y otra correspondiente al intestino medio (Fig. 2C). El tejido posterior del intestino anterior se observa como dos compartimentos separados y redondeados (Figs. 2C-2D), además, muestra células epiteliales columnares, y presenta músculos longitudinales y circulares internos que envuelven el epitelio externamente. Los núcleos ovales grandes son conspicuos dentro de las células epiteliales y musculares (Fig. 2D).

Intestino medio o mesenterón. Es un tubo simple, cuya longitud corresponde a la mitad del total del intestino en $B$. impatiens. Inicia en la región posterior del proventrículo, donde comienza la cardia, justo antes de la unión de los ciegos gástricos (Figs. 3A-3B). El intestino medio termina
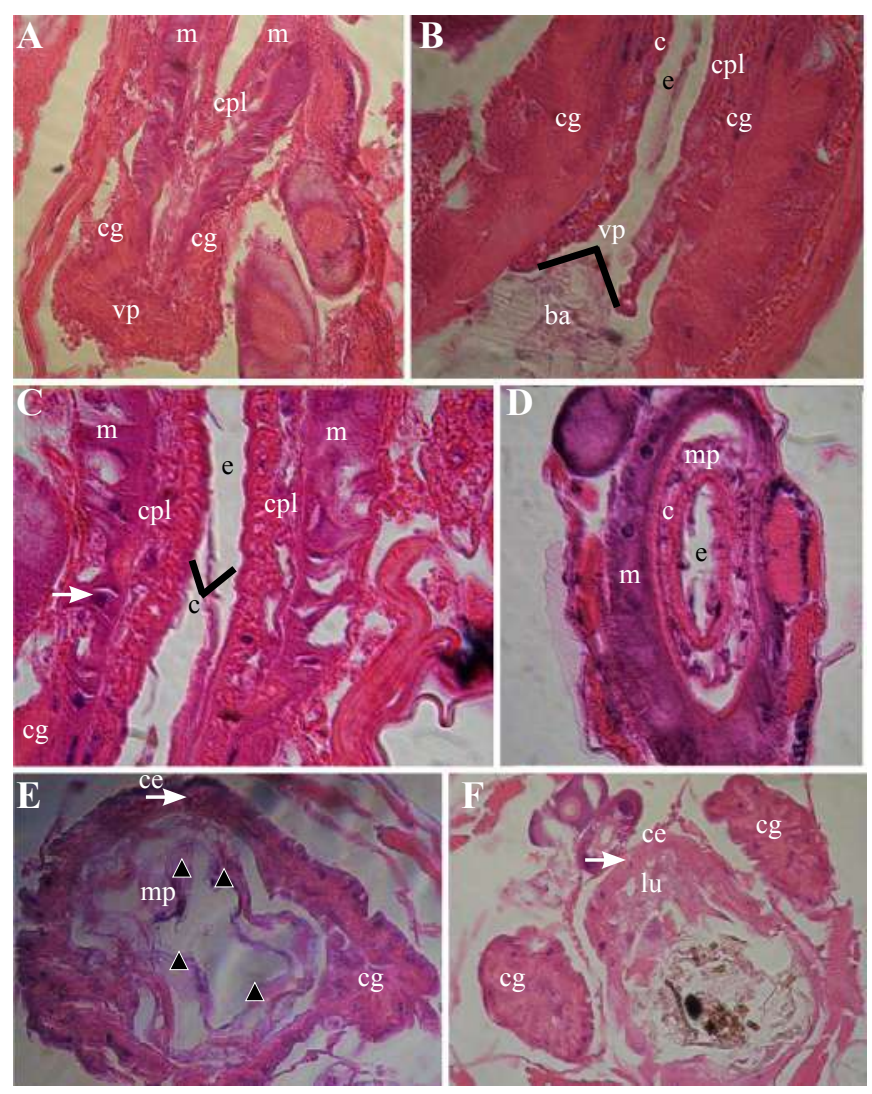

Figura 3. Proventrículo y cardia. A. Corte longitudinal de unión del intestino medio anterior e intestino medio, cardia con válvula del proventrículo cerrada $(40 x)$. B. Vista longitudinal de cardia con válvula del proventrículo abierta (100x). C. Cardia (40x). D. corte transversal de la cardia (40x). E. Corte transversal de unión de ciegos gástricos y la cardia, membrana peritrófica (40x). F. Unión de ciegos gástricos e intestino medio. Bolo alimenticio (ba), células epiteliales (ce) del intestino anterior (flecha blanca), células productoras de lámina (membrana peritrófica) (cpl), ciegos gástricos ( $\mathrm{cg}$ ), cutícula (c), esófago (e), lumen (lu), músculo $(\mathrm{m})$, membrana peritrófica ( $\mathrm{mp}$ ) (cabeza de flecha negra), válvula del proventrículo relajada (vp) (punta de flecha grande). donde se une con el píloro y los tubos de Malpighi. La cardia es un compartimiento redondeado, compuesto por células epiteliales. En el interior del lumen central de la cardia se observa una estructura plegada, que probablemente sea la MP (Figs. 3D-3F). Las células epiteliales de esta región tienen grandes núcleos ovales (Fig. 3C).

Las larvas de $B$. impatiens producen permanentemente la MP tipo II, la cual recorre todo el intestino medio hasta invaginarse en el píloro. Esta membrana divide al ventrículo en dos espacios endoperitrófico y ectoperitrófico (Fig. 3B).

Bradysia impatiens presenta dos ciegos gástricos bien desarrollados, que se insertan inmediatamente encima de donde se origina la MP. Los ciegos gástricos son de forma tubular, tan largos como las dos terceras partes del largo del intestino medio y cada uno de ellos tan anchos como la cuarta parte del intestino medio. Tanto los músculos internos circulares como los externos envuelven los ciegos gástricos. Las paredes internas del espacio ectoperitrófico están compuestas de células epiteliales cuboidales que forman un tubo y presentan un arreglo de forma ondulante (arriñonada) que hace lento el recorrido del líquido a través del lumen (Fig. 3E). Las características de los ciegos gástricos permiten suponer que en esta región es donde se realiza la mayor parte de la absorción de los nutrientes y del agua del intestino medio. El ciego gástrico se conforma por tres tipos de células: columnares, de regeneración y secretoras. La mayor parte de los núcleos de las células son grandes y ovales. Además, las células presentan microvellosidades que rodean el área de la célula que está en contacto con el lumen, lo que permite aumentar el espacio de absorción de nutrientes (Fig. 4E). Las células regenerativas se encontraron diseminadas por todo el epitelio, asociadas a las células columnares, lo que indica que el epitelio puede renovarse regularmente o bajo ciertas condiciones. En la parte anterior del intestino medio emerge de la cardia, su aspecto externo es similar al del ciego gástrico, en la superficie es muy lisa con los músculos internos circulares (Fig. 4A). El tejido del intestino medio anterior contiene MP dentro de su lumen central y está rodeado por una sola capa de células epiteliales cuboidales.

La parte media del mesenterón es una región más dilatada con respecto al proctodeo y al estomodeo (Fig. 4B) y su estructura es semejante a la del intestino medio anterior, con la MP dentro del lumen rodeado por una capa de células epiteliales cuboidales. La MP se presenta como una membrana fina y transparente (Fig. 4B), mientras que las células epiteliales del intestino medio contienen numerosos gránulos secretorios tanto interna como externamente (Fig. 4B). Además, las células del cuerpo graso también son evidentes entre estas células epiteliales y se proyectan hacia adentro desde sus membranas de base y cada una tiene microvellosidades largas que cubren sus superficies apicales internas. El intestino medio posterior está limitado por el píloro. En esta zona se acumula el material del bolo alimenticio no digerido por las otras regiones del intestino, empaquetándolo en segmentos para ser enviado al proctodeo. Sin embargo, la morfología de las células epiteliales es sinuosa y muestra gran cantidad de vellosidades largas, lo que sugiere que su función es de absorción de nutrientes y secreción (Figs. 4C-4D).

El intestino posterior (Proctodeo). De las L4 de B. impatiens está compuesto por el píloro, los túbulos de Malpighi, el íleon, el colon, el recto y el ano posterior. En el píloro se observó una capa única característica de células epiteliales cuboidales 
aplanadas, membrana peritrófica y capa muscular (Fig. 4F). También se observaron grandes gránulos secretores. Estos gránulos son similares a los encontrados en las células del intestino medio, pero que se presentan en menor cantidad y sólo aparecen en la región apical de las células pilóricas con núcleos relativamente grandes. Los túbulos de Malpighi emergen de la unión del intestino medio y el intestino posterior. Cada uno diverge distalmente en dos estructuras tubulares, formando los cuatro túbulos de Malpighi (Fig. 4F). Estos túbulos consisten en cadenas largas de células cuboidales. En un corte transversal se observa que las células contienen un núcleo grande, numerosos gránulos diminutos, microvellosidades cortas y abundantes en su superficie apical que está en contacto con el lumen (Fig. 4F). La superficie externa del íleon, colon, recto y del ano es similar a la del intestino medio al estar cubierta con una capa muscular e incrustada con abundantes traqueolas para el intercambio gaseoso (Figs. 5A-5B). Se observó una cutícula característica del intestino posterior, que rodeaba el lumen del íleon (Fig. 5A). En una sección transversal del íleon, se observa que su pared está formada principalmente por una capa de células epiteliales cuboidales con grandes núcleos ovalados y alguna fibra muscular periférica (Figs. 5A y 5B) y en esta región se observó la MP. Una sección del colon (Fig. 5C) y del recto (Fig. 5D) mostró tener características similares con el íleon,
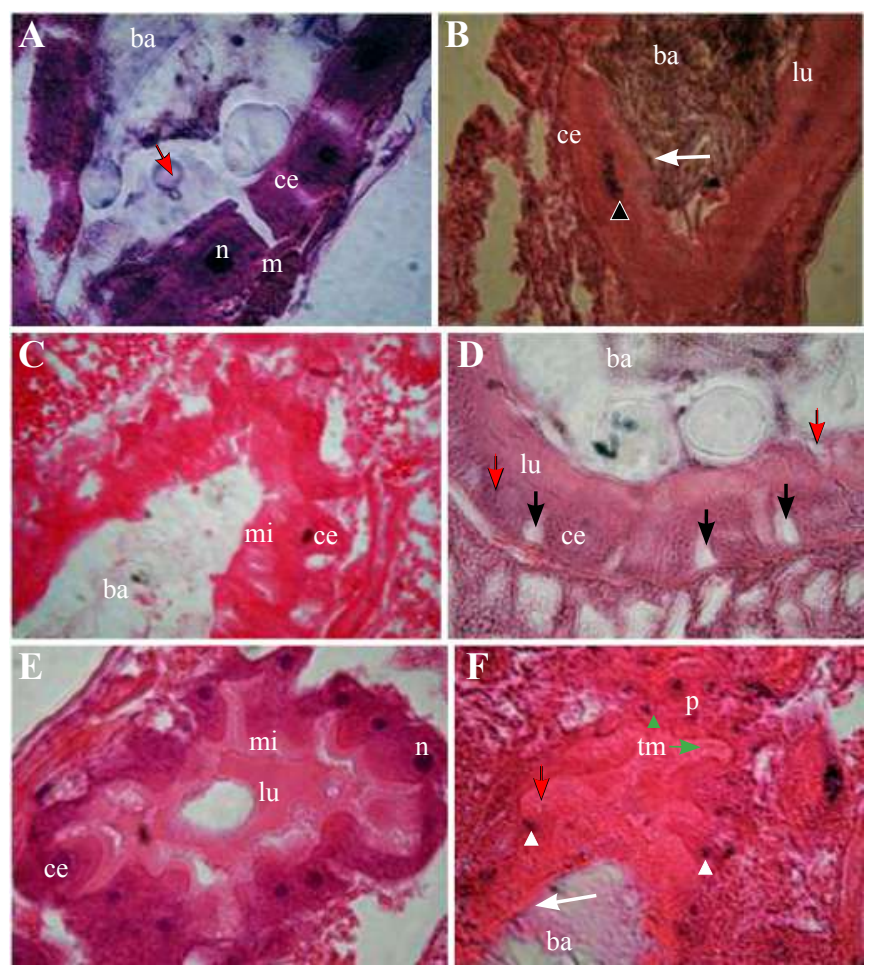

Figura 4. Intestino medio, ciegos gástricos y tubos de Malpighi (100x). A. Células epiteliales del intestino medio anterior. B. Células epiteliales del intestino medio medio. C y D. Células epiteliales del intestino medio posterior con abundantes microvellosidades. E. Vista transversal de ciego gástrico. F. Corte longitudinal de tubos de Malpighi. Bolo alimenticio (ba), células epiteliales (ce), lumen (lu), músculo (m), microvellosidades (mi), núcleo celular (n), píloro (p), tubos de Malpighi (tm), membrana peritrófica (punta de flechas blancas), gránulos secretores (flecha roja), células del cuerpo graso (flechas negras), núcleo celular (cabeza de flecha negra). núcleos células pilóricas (cabeza de flecha blanca), células cuboidales con núcleos grandes (cabeza de flecha verde), microvellosidades de las células de los tubos de Malpighi (flecha verde). excepto que exhibieron más fibra muscular que éste. En la región anal, las células mioepiteliales que son uninucleadas o multinucleadas comprenden el componente más grande de la pared del tubo anal (Fig. 5F). La MP se observa dentro del tubo anal (Fig. 5F).

pH del intestino medio y ciegos gástricos. El intestino medio en las L4 de $B$. impatiens, tiene un gradiente de $\mathrm{pH}$ que varía de 10-11 en el intestino medio anterior a 6,5-7,5 en el intestino medio posterior en la región del espacio endoperitrófico, mientras que en el espacio ectoperitrófico y los ciegos gástricos el pH es neutro (Fig. 6). Observaciones previas mostraron que el alimento pasa rápidamente por el intestino anterior y posterior, por lo cual no fue posible medir $\mathrm{el} \mathrm{pH}$ en estas regiones del tubo digestivo.

Recirculación del intestino medio. En $2 \mathrm{~h}$ el azul de dextran de $20 \mathrm{kDa}$ recorrió el tracto digestivo de las L4 de B. impatiens, aunque no se observó tinción en el intestino anterior, mientras que el intestino posterior se tiñó solo cuando el bolo alimenticio era empujado en forma de excretas. Sin embargo, en el intestino medio el espacio endoperitrófico se comenzó a teñir a los 15 min y después de 30 min quedó teñido por completo; el espacio ectoperitrófico comenzó a teñirse a los $45 \mathrm{~min}$ y los ciegos gástricos a los $60 \mathrm{~min}$. Se observó que en las larvas de $B$. impatiens existe un mecanismo de recirculación del bolo alimenticio en todo el intestino medio (espacio endo y ecto peritrófico). Dependiendo del tamaño de las fracciones alimenticias pueden atravesar la MP y entrar al espacio ectoperitrófico y pasar a formar parte del líquido del lumen, el cual recorre el intestino medio en dirección del píloro a los ciegos gástricos. Posteriormente, el líquido del lumen recorre los ciegos gástricos y las fracciones que no son asimiladas regresan nuevamente al espacio endoperitrófico, para recircularse o pasar al intestino posterior para ser evacuado (Fig. 6).

Permeabilidad de la membrana peritrófica. La MP en las L4 de B. impatiens fue permeable al azul de dextran de 5, $10 \mathrm{y}$ $20 \mathrm{kDa}$, una hora después de su aplicación. En todos los casos el espacio endoperitrófico se tiño de azul en $30 \mathrm{~min}$, la tinción del espacio ectoperitrófico y los ciegos gástricos a los $60 \mathrm{~min}$. A las $24 \mathrm{~h}$ el azul de dextran de $500 \mathrm{kDa}$ se encontró en el espacio endoperitrófico y después de $48 \mathrm{~h}$ el azul de dextran permaneció en el espacio endoperitrofico. Esto indica que la MP es impermeable a este tamaño de molécula.

\section{Discusión}

El tracto digestivo de las L4 de B. impatiens presentó una estructura similar a la mayoría de los insectos dividiéndose en tres regiones: estomodeo, mesenteron y proctodeo (Chapman 1998; Terra y Ferreira 2012). En las L4 no fue posible identificar un divertículo (buche), lo que indica que no hay almacenamiento de alimento en el estomodeo. Además, con la tinción del bolo alimenticio no se observó que permaneciera almacenado en algún órgano, sino que pasa de manera inmediata al intestino medio, lo que también permite considerar que en las larvas de $B$. impatiens no existe el buche. Esta característica también se presenta en las larvas de cuarto estadio de L. longipalpis (Do Vale et al. 2007). En el orden Diptera, el buche es una estructura que tiene la función de almacenamiento y digestión del flujo del bolo alimenticio 

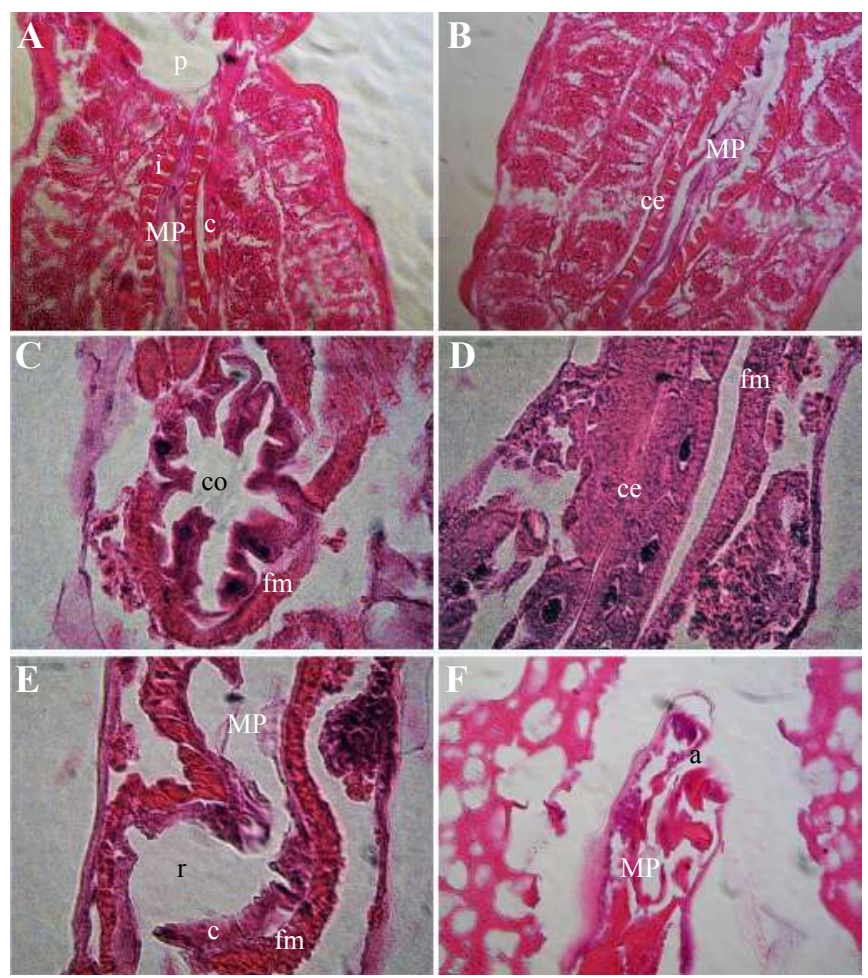

Figura 5. Intestino posterior (proctodeo). A. Vista longitudinal del píloro e íleon (40x). B. Corte longitudinal del íleon (100x). C. Vista transversal colon (100x). D. Vista longitudinal del recto (40x). E y F. Vista longitudinal del recto y ano (100x y 40x, respectivamente). Ano (a), células epiteliales (ce), colon (co), cutícula (c), íleon (i), membrana peritrófica (MP), píloro (p), recto (r), fibra muscular (fm).

(Stoffolano y Halselton 2013). Las células del estomodeo no intervienen en el proceso de secreción de enzimas o absorción de nutrientes, característica que es similar a la de otros insectos (Terra y Ferreira 2012). La cutícula (epi- y endocutícula) que se observó en el esófago de la L4 de $B$. impatiens fue estructuralmente similar al de la mosca $C$. megacephala (Boonsriwong et al. 2006).

Las características morfológicas de las glándulas salivales de B. impatiens se asemejan a las mostradas por Evangelista y Leite (2007) para la larva de primer estadio de Dermatobia hominis (L., 1781) (Diptera: Oestridae) y C. megacephala (Boonsriwong et al. 2006), que tienen glándulas tubulares simples que se abren en estrechos conductos eferentes en cada lado, pero $B$. impatiens difieren en que convergen para formar un solo conducto deferente mediano que conduce a la cavidad oral.

La cardia es el órgano donde se encuentran las células que producen la MP, la cual es del tipo II y distingue al orden Diptera (Hegedus et al. 2009). Las L4 de B. impatiens poseen un proventrículo globoso Esta estructura también se observa en Stomoxys calcitrans (L., 1758) (Diptera: Muscidae) (Lehane 1997) o en la mosca de la fruta, Bactrocera dorsalis (Hendel, 1912) (Diptera: Tephritidae) (Lee et al. 1998); C. megacephala (Boonsriwong et al. 2006) y en L. longipalpis (Do Vale et al. 2007). Además, se observó que la MP es producida de forma continua por la cardia y se distribuye a lo largo del intestino medio (Terra y Ferreira 2012).

Las L4 de B. impatiens presentan dos ciegos gástricos. El número de ciegos gástricos en las larvas de los dípteros puede variar de cero a ocho y así, en Gasterophilus intestinalis (De

Geer, 1776) (Diptera: Oestridae) (Roelfstra et al. 2010) y $L$. longipalpis (Diptera: Psychodidae) (Do Vale et al. 2007) no están presentes. En Rhynchosciara americana (Wiedemann, 1821) (Diptera: Sciaride) se encuentran dos (Ferreira et al. 1981), mientras que, Odontosciara (Diptera: Sciaridae) (Cruz y Morelli 1985) y C. megacephala tienen cuatro (Boonsriwong et al. 2006) y las larvas de "mosca negra" (Diptera: Simuliidae) de cero a ocho (Kim y Adler 2009). El análisis morfológico de los ciegos gástricos en las larvas de B. impatiens, muestra que tienen mayor volumen con respecto a las larvas de las especies de dípteros antes mencionadas, característica que, junto con la forma arriñonada de las células epiteliales y la gran cantidad de microvellosidades apicales y laterales de las células columnares, permiten suponer que en esta región se realiza la mayor absorción de nutrientes. La presencia de microvellosidades en las células es indicativa de que su función es de absorción (Cruz y Morelli 1985). Además, los ciegos gástricos contribuyen de forma importante para crear el flujo de contracorriente en los espacios endo y ectoperitrófico (Terra et al. 1988; Terra 1990), el mecanismo de flujo de contracorriente permite reciclar las enzimas que intervienen en el proceso de la digestión. En muchas de las larvas del suborden Nematocera, la excreción del agua del intestino medio se realiza en la parte posterior de los ciegos gástricos (Terra et al. 1988). La tinción del intestino medio por el azul de dextran de $20 \mathrm{kDa}$ mostró que el agua entra a través del tracto digestivo y del intestino medio posterior, saliendo por los ciegos gástricos y reingresa al espacio ectoperitrófico por alguna región del intestino medio posterior o por los tubos de Malpighi. Proceso que fue descrito por Dow (1981) y sugiere que algunas de las funciones de los ciegos gástricos en las larvas de B. impatiens es la de absorción de nutrientes y recirculación del bolo alimenticio. Sin embargo, es necesario realizar estudios más detallados sobre este mecanismo en los estadios inmaduros de esta especie de Sciaridae.

En las larvas de B. impatiens el intestino medio está divido en dos regiones por la MP: espacio endoperitrófico y ectoperitrófico. Esta organización es característica de los dípteros como Aedes aegypti (L., 1762) (Diptera: Culicidae) (Ferreira et al. 2010), Culex quinquefaciatus (Say, 1823) (Diptera: Cilicidae) (Nunes et al. 2010) y C. megacephala (Boonsriwong et al. 2006). En el presente trabajo se evidenció que la MP de las L4 vivas de B. impatiens es permeable a moléculas iguales o menores de $20 \mathrm{kDa}$ y es impermeable a moléculas de $500 \mathrm{kDa}$, resultados que son similares a los obtenidos en larvas de $R$. americana, en las cuales la MP es permeable a moléculas menores de $100 \mathrm{kDa}$ (Bolognesi et al. 2008), en A. aegypti y Anopheles gambiae (Giles, 1902) (Diptera: Culididae) la MP es permeable a moléculas iguales o menores a $148 \mathrm{kDa}$ (Edwards y Jacobs-Lorena 2000). El paso de moléculas de hasta $148 \mathrm{kDa}$ indica que la MP en larvas L4 de B. impateins tiene poros de hasta $9 \mathrm{~nm}$ (Edwards y Jacobs-Lorena 2000). El tamaño de los poros de la MP en las larvas de los dípteros tiene un rango de 7.5-9 $\mathrm{nm}$ de diámetro (Terra y Ferreira 1983; Miller y Lehane 1990; Edwards y Jacobs-Lorena 2000; Terra 2001; Bolognesi et al. 2008). Este tamaño de poro sugiere que la MP es permeable a proteínas globulares de aproximadamente $148 \mathrm{kDa}$. Nuestros resultados son consistentes con el hecho de que macromoléculas como las enzimas digestivas deben atravesar la MP para realizar la digestión del bolo alimenticio.

Las larvas de B. impatiens se han asociado con el transporte de hongos fitopatógenos (Marín-Cruz et al. 2015a), debido 


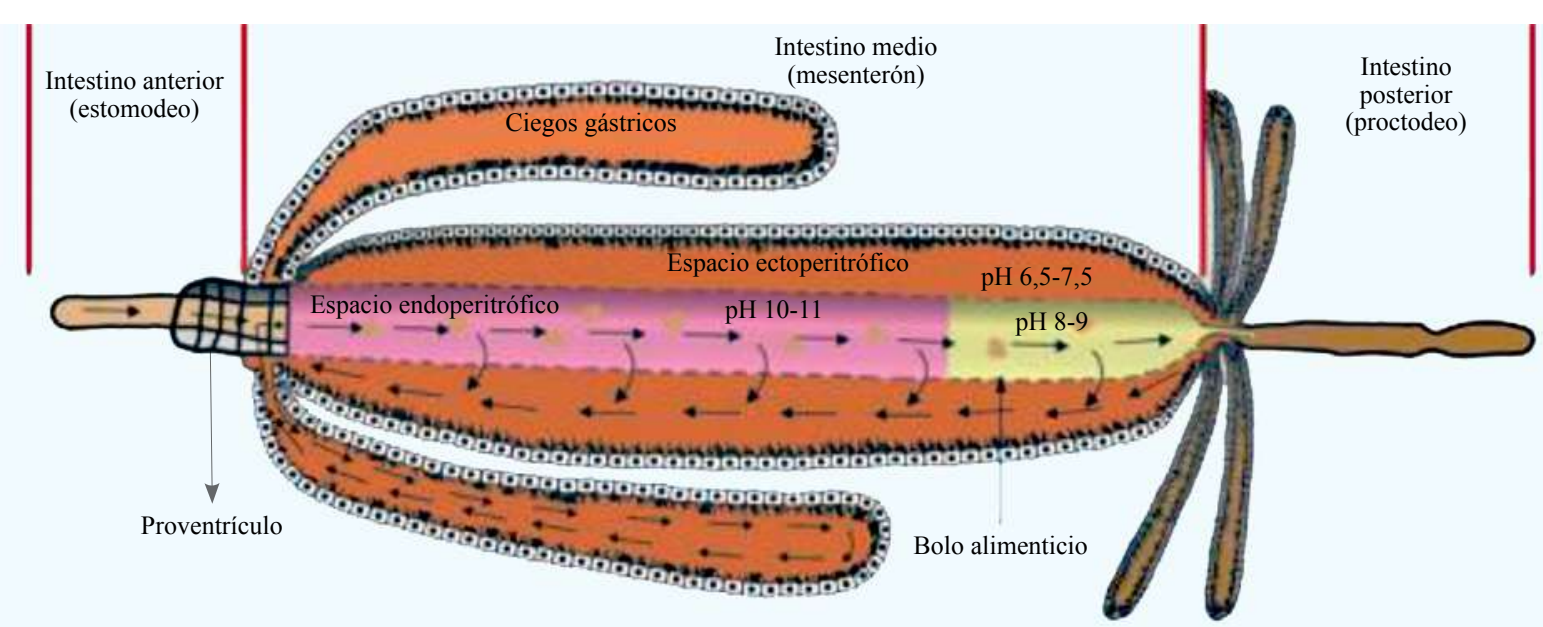

Figura 6. Esquema del sistema de recirculación y pH en el intestino medio en larvas de cuarto instar de Bradysia impatiens. Ilustración de Victor Hugo Marín-Cruz en 2018.

a que los conidios pasan por el tracto digestivo sin sufrir daño alguno en su capacidad de germinación lo que podría indicar que el tamaño del poro de la MP impide el paso de los conidios al espacio ectoperitrófico, aunque se requiere de un estudio más detallado.

El intestino medio es la región responsable de la digestión y la absorción (Dow 1986). El presente estudio muestra que el intestino medio de la L4 B. impatiens está dividido en las regiones anterior, media y posterior, como se describió para larvas de G. intestinalis (Roelfstra et al. 2010). Se observaron tres tipos de células: epiteliales, regenerativas y endocrinas, presentes también en la L4 de O. togoi (Intakhan et al. 2014). Las células epiteliales que constituyen el intestino medio anterior son columnares aplanadas, sin aumento del área que está en contacto con el lumen y su función parece ser de unión de los ciegos gástricos con el intestino medio, además de que presentan microvellosidades cortas. Las células columnares que constituyen el epitelio del intestino medio y posterior tienen microvellosidades largas en la membrana plasmática apical y, por lo tanto, el área de la membrana disponible para la secreción o absorción se incrementa. La membrana plasmática basal tiene numerosos pliegues, que forman un laberinto de canales complejo, están generalmente relacionadas con el transporte activo de agua e iones (Terra et al. 1988) y pueden estar involucradas en flujos de intestino medio importantes para la translocación de enzimas y productos de la digestión (Terra y Ferreira 2005). Las células regenerativas en larvas de B. impatiens son similares a las reportadas por Evangelista y Leite (2007) en larvas de D. hominis, pero según Terra et al. (1988) parecen estar ausentes en algunas larvas de dípteros. El papel de las células endócrinas en el control de las funciones del intestino medio aún no está claro, pero en los vertebrados regulan actividades intestinales (Lehane 1997; Boonsriwong et al. 2006; Taha et al. 2010).

El $\mathrm{pH}$ del intestino medio es una propiedad ambiental interna que afecta la actividad de las enzimas digestivas y es el resultado de la adaptación de un insecto ancestral a una dieta particular (Terra y Ferreira 2012). Por lo regular, las larvas de dípteros detritívoros de Nematocera tienen un gradiente de $\mathrm{pH}$ en el intestino medio, con una alta alcalinidad en el intestino medio anterior y bajo nivel del pH en la región del intestino medio posterior, característica que ha sido reportada en L. longipalpis (Do Vale et al. 2007), A. gambiae
(Neira et al. 2008), C. quinquefasciatus (Nunes et al. 2010) y T. abdominalis (Martin et al. 1980). El pH en la L4 de $B$. impatiens, presenta un comportamiento similar al de las larvas de Nematocera, por lo que la función de cada región del intestino medio de $B$. impatiens, puede ser similar a la que reportan Neira et al. (2008) para larvas de A. gambiae, donde los ciegos gástricos y el intestino medio posterior tienen una función de metabolismo de proteínas y carbohidratos y en menor medida el metabolismo y absorción de lípidos. Ferreira y Terra (1982), al analizar la actividad enzimática específica en diferentes regiones del intestino medio de larvas del siárido detritívoro R. americana, que se alimenta de hongos y materia orgánica, sugirieron que los ciegos gástricos y el intestino medio posterior son los principales compartimentos donde se produce la digestión de proteínas y carbohidratos, con poca o ninguna participación del intestino medio anterior y señalaron que en las larvas de los dípteros, los ciegos gástricos son particularmente ricos en peptidasas, mientras que la actividad de carbohidrasas fue más abundante en el intestino medio posterior.

Los altos niveles de alcalinidad del intestino medio anterior están relacionados con la solubilización de complejos proteína-tanino, o la extracción de hemicelulosa del detritus foliar ingerido (Terra 1990). Además, la alta alcalinidad del intestino medio anterior puede actuar en las partículas de humus y en los microorganismos presentes en la dieta de la larva, lo que permite la solubilización y dispersión más fácil y rápida de proteínas, carbohidratos complejos y lípidos y facilita la acción de las enzimas digestivas (Terra 1988). Neira et al. (2008) reportan que el $\mathrm{pH}$ tan alcalino en el intestino medio sugiere que la absorción de lípidos es intensa en esta región, ya que las condiciones alcalinas en el intestino de insectos podrían reducir la precipitación de sustancias surfactantes como la lisolecitina, presentes en el fluido intestinal, lo que a su vez facilita la solubilización de lípidos en la dieta y el transporte a través de las células de la mucosa (De Veau y Schultz 1992).

\section{Conclusiones}

El tracto digestivo de larvas de cuarto instar de $B$. impatiens se divide en mesenterón, estomodeo y proctodeo. Las células que lo componen son columnares, regenerativas y endocrinas. 
La digestión del bolo alimenticio se realiza en el intestino medio, tienen dos ciegos gástricos bien desarrollados, donde existe una gran absorción de nutrientes. La MP es del tipo II, con un tamaño de poro que permite el paso de moléculas de hasta $150 \mathrm{kDa}$. Tiene cuatro tubos de Malpighi. El intestino medio tiene un gradiente de $\mathrm{pH}$ que va de alcalino (intestino anterior) a neutro (ciegos gástricos e intestino medio posterior). Presenta un mecanismo de recirculación que mejora la digestión y optimiza la producción de enzimas que van del espacio endoperitrófico al ectoperitrófico y viceversa. Las características morfofisiológicas mostradas por las larvas permiten confirmar que son organismos bien adaptados como detritívoros.

\section{Agradecimientos}

Los autores agradecen: Osvaldo López Díaz, responsable del laboratorio de Histopatología perteneciente a la Licenciatura de Medicina Veterinaria y Zootecnia de la UAM-X; Celina Llanderal Cázares por su valiosa aportación como revisora; al Consejo Nacional de Ciencia y Tecnología (CONACYT) por la beca de estudios de doctorado otorgada a Víctor Hugo Marín Cruz, y a la Universidad Autónoma Metropolitana Unidad Xochimilco y al Doctorado en Ciencias Agropecuarias (UAM-X) por el financiamiento para desarrollar el presente trabajo.

\section{Literatura citada}

BOLOGNESI, R.; TERRA, W. R.; FERREIRA, C. 2008. Peritrophic membrane role in enhancing digestive efficiency theoretical and experimental models. Journal of Insect Physiology 54: 14131422. https://doi.org/10.1016/j.jinsphys.2008.08.002

BOONSRIWONG, W.; SUKONTASON, K.; OLSON, J. K.; VOGTSBERGER, R. C.; CHAITHONG, U.; KUNTALUE, B.; NGERN-KLUN, R.; UPAKUT, S.; SUKONTASON, K. L. 2006. Fine structure of the alimentary canal of the larval blow fly Chrysomya megacephala (Diptera: Calliphoridae). Parasitology Research 100 (3): 561-574. https://doi.org/10.1007/s00436-0060363-0

CHAPMAN, R. F. 1998. The insects: structure and function, 4th ed. Cambridge University Press, Cambridge, Reino Unido.770 p.

CIBRIÁN, T. D.; GARCÍA, D. S.; DON JUAN, M. B. 2008. Manual de identificación y manejo de plagas y enfermedades en germoplasma y planta producida en viveros. Comisión Nacional Forestal. México. 153 p.

CRUZ, L. C. da; MORELLI S. M. R. L. 1985. Aspectos da ultraestructura dos cecos gástricos da larva de Odontosciara sp. (Diptera: Sciaridae). Revista Brasileira de Zoologia 3 (4): 181188. https://doi.org/10.1590/S0101-81751985000400004

DE VEAU, E. J. I.; SCHULTZ, J. C. 1992. Reassessment of interaction between gut detergents and tannins in Lepidoptera and significance for gypsy-moth larvae. Journal of Chemical Ecology 18 (8): 1437-1453. https://doi.org/10.1007/ BF00994367

DO VALE, F. V.; PEREIRA, H. M.; FIGUEIREDO, N. G. 2007. Midgut $\mathrm{pH}$ profile and protein digestion in the larvae of Lutzomyia longipalpis (Diptera: Psychodidae). Journal of Insect Physiology 53 (11): 1151-1159. https://doi.org/10.1016/j. jinsphys.2007.06.005

DOW, J .A. T. 1981. Countercurrent flows, water movements and nutrient absorption in the locust midgut. Journal of Insect Physiology 27 (9): 579-585. https://doi.org/10.1016/00221910(81)90104-9

DOW, J. A. T. 1986. Insect midgut function. pp. 187-328. En: Evans, P. D.; Wigglesworth, V. B. (Eds.). Advances in Insect Physiology.
Volumen 19: University Cambrige, Academic Press. London. England. 434 p. https://doi.org/10.1016/S0065-2806(08)60102-2

EVANGELISTA, L. G.; LEITE, A. C. 2007. Salivary glands of second and third instars of Dermatobia hominis (Diptera: Oestridae). Journal of Medical Entomology 44 (3): 398-404.

EDWARDS, M. J.; JACOBS-LORENA, M. 2000. Permeability and disruption of the peritrophic matrix and caecal membrane from Aedes aegypti and Anopheles gambiae mosquito larvae. Journal of Insect Physiology 46 (9): 1313-1320. https://doi.org/10.1016/ S0022-1910(00)00053-6

FERREIRA, C.; TERRA, W. R. 1982. Function of midgut ceca and ventriculus microvilli bound enzymes from cells of different midgut regions of starving and feeding Rhynchosciara americana larvae. Insect Biochemistry 12 (3): 257-262. https:// doi.org/10.1016/0020-1790(82)90083-X

FERREIRA, C.; RIBEIRO, A. F.; TERRA, W. R. 1981. Fine structure of the larval midgut of the fly Rhynchosciara and its physiological implications. Journal Insect Physiology 27 (8): 559-570. https://doi.org/10.1016/0022-1910(81)90044-5

FERREIRA, C.; CAVASIN, G.; GARCIA, S. H. H.; GERIS, R.; GARCIA, S. I. 2010. Alterações morfo-histológicos em larvas de Aedes aegypti (Linnaeus, 1762) (Diptera: Culicidae) causadas pelo tanino catéquico isolado da plantado cerrado Magonia pubescens (Sapindaceae). Revista Patología Tropical 39 (4): 309-321. https://doi.org/10.5216/rpt.v39i4.13066

GONTIJO, N. F.; ALMEIDA-SILVA, S.; COSTA, F. F.; MARESGUIA, M. L.; WILLIAMS, P.; MELO, M. N. 1998. Lutzomyia longipalpis: $\mathrm{pH}$ in the gut, digestive glycosidases and some speculations upon Leishmania development. Experimental Parasitology 90 (3): 212-219. https://doi.org/10.1006/ expr.1998.4336

HEGEDUS, D.; ERLANDSON, M.; GILLOT, C.; TOPRAK, U. 2009. New insights into peritrophic matrix synthesis, architecture, and function. Annual Review of Entomology 54: 285-302. https:// doi.org/10.1146/annurev.ento.54.110807.090559

HURLEY, B. P.; SLIPPERS, B.; WINGFIELD, B. D.; GOVENDER, P.; SMITH J. E.; WINGFIELD, M. J. 2010. Genetic diversity of Bradysia difformis (Sciaridae: Diptera) populations reflects movement of an invasive insect between forestry nurseries. Biological Invasions 12 (4): 729-733. https://doi.org/10.1007/ s10530-009-9509-1

INTAKHAN, N.; JARIYAPAN, N.; CHANMOL, W.; SOR-SUWAN, S.; PHATTANAWIBOON, B.; SAEUNG, A.; CHOOCHOTE, W.; BATES, P. A. 2014. Midgut ultrastructure of fourth instar Ochlerotatus togoi (DIPTERA: CULICIDAE). Southeast Asian Journal of Medicine and Public Health 45 (6): 1301-1315.

JAMES, L. N. 2002. Insect physiology and biochemistry. CRS Press. Washington D.C., EE. UU. 485 p.

KIM, S. K.; ADLER, P. H. 2009. Digestive system of larval black flies (Diptera: Simuliidae): structure and phylogeny. Invertebrate Biology 128 (2): 182-204. https://doi.org/10.1111/j.17447410.2008.00161.X

LEE, W. Y.; CHEN, M. E.; LIN, T. L. 1998. Morphology and ultrastructure of the alimentary canal of Oriental fruit fly Bactrocera dorsalis (Hendel) (Diptera: Tephritidae) (I): the structure of the foregut and cardia. Zoological Studies 37 (2): 95-101.

LEHANE, M. J. 1997. Peritrophic matrix structure and function. Annual Review of Entomology 42: 525-550. https://doi. org/10.1146/annurev.ento.42.1.525

MARIIN-CRUZ, V. H.; HUERTA, H.; RODRÍGUEZ-NAVARRO, S. 2017. Familia Sciaridae. pp. 444-446. En: Cibrian-Tovar, D. (Ed.). Fundamentos de entomología forestal. Universidad Autónoma Chapingo. Texcoco, Estado de México, México. 531 p.

MARÍN-CRUZ, V. H.; CIBRIÁN-TOVAR, D.; MÉNDEZMONTIEL, J. T.; PÉREZ-VERA, O. A.; CADENA-MENESES, J. A. 2015a. Control del mosco fungoso negro Lycoriella ingenua (Dufour, 1839) y Bradysia impatiems (Johannsen, 1912) (Diptera: Sciaridae) en Pinus montezumae Lamb. Revista 
Mexicana Ciencias Forestales 6 (27): 90-100. https://doi. org/10.29298/rmcf.v6i27.283

MARIN-CRUZ, V. H.; CIBRIÁN-TOVAR, D.; MÉNDEZMONTIEL, J. T.; PÉREZ-VERA, O. A.; CADENA-MENESES, J. A.; HUERTA, H.; RODRÍGUEZ-YAM, G.; CRUZRODRÍGUEZ, J. A. 2015b. Biología de Lycoriella ingenua y Bradysia impatiens (Diptera: Sciaridae). Madera y Bosques 21 (1): 113-128. https://doi.org/10.21829/myb.2015.211436

MARTIN, M. M.; MARTIN, J. S.; KUKOR, J. J.; MERRITT, R. W. 1980. The digestion of protein and carbohydrate by the stream detritivore, Tipula abdominalis (Diptera: Tipulidae). Oecologia 46 (3): 360-364. https://doi.org/10.1007/BF00346265

MARTÍNEZ, M. I. 2002. Técnicas básicas de anatomía microscópica y de morfometría para estudiar a los insectos. Boletín de la Sociedad Entomológica Aragonesa 30: 187-195.

MENZEL, F.; SMITH, J. E.; CHANDLER, J. P. 2006. The sciarid fauna of the British Isles (Diptera: Sciriadae), including descriptions of six new species. Zoological Journal of the Linnean Society 146 (1): 1-147. https://doi.org/10.1111/j.10963642.2006.00190.x

MILLER, N.; LEHANE, M. S. 1990. In vitro perfusión studies on the peritophic membrane of the Tsetse fly Glossina morsitans morsitans (Diptera: Glossinidae). Journal Insect Physiology 36 (11): 813-818. https://doi.org/10.1016/0022-1910(90)90167-E

MOHRIG, W.; MENZEL. F. 2009. Sciaridae (Black fungus gnats). pp. 279-292. En: Brown, B. V.; Borkent, A.; Cumming, J. M.; Wood, D. M.; Woodley N. E.; Zumbado, M. A. (Eds.). Manual of Central American Diptera Vol 1. Monograph Publishing Program. Canada: National Research Council of Canada. $714 \mathrm{p}$.

MOHRIG, W.; HELELR, K.; HIPPA, H.; VILKAMAA, P.; MENZEL, F. 2012. Revision of black fungus gnats (Diptera: Sciaridae) of North America. Studia Dipterologica 19 (1/2): 141-286.

NEIRA, O. M.; VANEKERIS, L.; CORENA-MCLEOD, M. D. P.; LINSER, P. J. 2008. A microarray-based analysis of transcriptional compartmentalization in the alimentary canal of Anopheles gambiae (Diptera: Culicidae) larvae. Insect Molecular Biology 17 (1): 61-72. https://doi.org/10.1111/j.13652583.2008.00779.x

NUNES, A. S.; SERRÃO, J. E.; LANE, M. A. 2010. Alterations in the fat body and midgut of Culex quinquefasciatus larvae following exposure to different insecticides. Micron 41 (6): 592597. https://doi.org/10.1016/j.micron.2010.04.004

ROELFSTRA, L.; VLIMANT, M.; BETSCHART B.; PFISTER, K.; DIEHL, P. A. 2010. Light and electron microscopy studies of the midgut and salivary glands of second and third instars of the horse stomach bot, Gasterophilus intestinalis. Medical and Veterinary Entomology 24 (33): 236-249. https://doi.org/10.1111/j.13652915.2010.00881.x

SHARMA, B. R.; MARTIN, M. M.; SHAFER, J. A. 1984. Alkaline proteases from the gut fluids of detritus feeding larvae of the crane fly Tipula abdominalis (Say) (Diptera: Tipulidae). Insect Biochemistry 14 (1): 37-44. https://doi.org/10.1016/00201790(84)90081-7

SHIN, S. G.; LEE, H. S.; LEE, S. 2012. Dark winged fungus gnats (Diptera: Sciaridae) collected from shiitake mushroom in Korea. Journal of Asia-Pacific Entomology 15 (1): 174-181. https://doi. org/10.1016/j.aspen.2011.09.005

STOFFOLANO, G. J.; HASELTON, A. 2013. The adult dipteran crop: a unique and overlooked organ. Annual Review of Entomology 58: 205-225. https://doi.org/10.1146/annurevento-120811-153653
TAHA, N.; ABDEL-MEGUID, A.; EL-EBIARIE, A.; TOHAMY, A. A. 2010. Ultrastructure of the midgut of the early third larval instar of Chrysomya megacephala (Diptera:Calliphoridae). Journal of American Science 6 (10): 1-6.

TERRA, W. R. 1990. Evolution of digestive systems of insects. Annual Review of Entomology 35: 181-200. https://doi. org/10.1146/annurev.ento.35.1.181

TERRA, W. R. 2001. The origin and funtions of the insect peritrophic membrane and peritrophic gel. Archives of Insect Biochemistry and Physiology 47: 47-61. https://doi.org/10.1002/arch.1036

TERRA, W. R.; FERREIRA, C. 1983. Further evidence that enzimes involved in the final stages of digestion by Rhynchosciara americana do not enter the endoperitrophic space. Insect Biochemistry 13 (2): 143-150. https://doi.org/10.1016/00201790(83)90077-X

TERRA, W. R.; FERREIRA, C. 2005. Biochemistry of digestion. pp. 171-224. En: Gilbert, L. I.; Iatrou, K.; Gill, S. S. (Eds.). Comprehensive Molecular Insect Science. Elsevier, Oxford. 560 p. https://doi.org/10.1016/B0-44-451924-6/00053-3

TERRA, W. R.; FERREIRA, C. 2012. Molecular and evolutionary physiology of insect digestion. pp. 93-115. En: Panizzi, A. R.; Parra, R. P. (Eds.). Insect bioecology and nutrition for integrated pest management. Taylor \& Francis Group, LLC. Estados Unidos de América. 732 p. https://doi.org/10.1201/b11713-6

TERRA, W. R.; ESPINOZA-FUENTES, F. P., RIBEIRO, A. F.; FERREIRA, C. 1988. The larval midgut of the housefly (Musca domestica): ultrastructure, fluid fluxes and ion secretion in relation to the organization of digestion. Journal of Insect Physiology 34 (6): 463-472. https://doi.org/10.1016/00221910(88)90187-4

URÍA, E.; MORA, C. 1996. Apuntes para el curso teórico práctico de histología animal. ENCB. IPN. México. 277 p.

\section{Origen y financiamiento}

Este trabajo se realizó dentro del proyecto de Doctorado en Ciencias Agropecuarias de la Universidad Autónoma Metropolitana Unidad Xochimilco. El cual fue financiado por el Consejo Nacional de Ciencia y Tecnología (CONACyT) con la beca número 258065 durante el periodo 2014-2018.

\section{Contribución de los autores}

Victor Hugo Marin-Cruz: Trabajo de laboratorio, cría de larvas de B. impatiens, cortes histológicos, identificación anatómica e histológica, investigación de literatura, escritura y discusión del manuscrito. Toma de fotografias. Realización de esquema de recirculación del bolo alimenticio.

Silvia Rodríguez-Navarro: Capacitación en cortes histológicos, apoyo en identificación de estructuras anatómicas; discusión y revisión de manuscrito.

Juan Esteban Barranco-Florido: Capacitación en técnicas de fijación de tejidos y tinción histológica, manejo de moléculas para determinar el tamaño de poro, medición de $\mathrm{pH}$ de la membrana peritrófica. Discusión y revisión de manuscrito.

Roberto Alejandro Terrón-Sierra: Técnicas de cría de B. impatiens, escritura, discusión y revisión manuscrito. Apoyo de equipo de fotografia.

David Cibrián-Tovar: Escritura del manuscrito, discusión y revisión del manuscrito. Apoyo con literatura, equipo de fotografía. 